\title{
ON AN APPLICATION OF A VARIANT OF THE CLOSED GRAPH THEOREM AND THE SECANT METHOD
}

\author{
IOANNIS K. ARGYROS
}

\begin{abstract}
The method of nondiscrete mathematical induction is used to find error bounds for the Secant method. We assume only that the operator has Hölder continuous derivatives. In the case the Fréchetderivative of the operator satisfies a Lipschitz condition our results reduce to the ones obtained by F. Potra (Num. Math. 1982).
\end{abstract}

\section{Introduction}

Consider the equation

$$
f(x)=0
$$

where $f$ is a nonlinear operator mapping a subset $E_{f}$ of a Banach space $E_{1}$ into another Banach space $E_{2}$.

Here we are concerned with finding solutions of (1) using the secant iterations

$$
\begin{aligned}
& x_{n+1}=x_{n}-\delta f\left(x_{n-1}, x_{n}\right)^{-1} f\left(x_{n}\right) \\
& x_{n+1}=x_{n}-\delta f\left(x_{-1}, x_{0}\right)^{-1} f\left(x_{n}\right)
\end{aligned}
$$

where $x_{-1}$ and $x_{0}$ are two points in the domain of $f$, and $\delta f$ is a consistent approximation of $f^{\prime}$.

Received April 11, 1990; revised March 16, 1993.

(1980) A.M.S. classification codes: 65J15, 65B05, 65L50, 65M50, $46 \mathrm{~B} 15$.

Key words and phrases: Nondiscrete mathematical induction, Secant method, Banach space, Hölder continuous derivatives. 
This work is based upon the elegant work of F. Potra included in [5] concerning the error analysis of the Secant method. One of Potra's basic assumptions is the fact that essentially the linear operator $f^{\prime}$ is Lipschitz continuous. However, in the presence of some interesting examples (see part (III)), where $f^{\prime}$ is only Hölder continuous, we extend most of the results contained in [5] for the iteration (2). We leave the extension of the results for (3) to the motivated reader.

We furnish two examples in part (III) to show that our results can be applied. where as the equivalent results in [5] cannot.

Since our results are drawn almost in the same lines with the ones in [5], we will need to restate some here.

Note that the results of this paper could be obtained by using one dimensional rate of convergence only, as was done in Potra [5], and not the more general rates of convergence introduced in Potra [6].

\section{Preliminaries}

Consider a class $C$ of pairs $\left(f, v_{0}\right)$ where $f$ is as above and $v_{0}=\left(x_{-k+1}, \cdots\right.$, $\left.x_{0}\right)$ is a system of $k$ points from $E_{f}$. We want to attach to each pair $\left(f, v_{0}\right) \in C$ a sequence $\left\{x_{n}\right\}, n=0,1,2, \cdots$ of points of $E_{f}$ converging to a root $x^{*}$ of (1). To achieve this we associate with the pair $\left(f, v_{0}\right)$ an operator $F: E \subset E_{f}^{p} \rightarrow E_{1}$, where $k \geq p$ and try to obtain a sequence $\left\{x_{n}\right\}, n=0,1,2, \cdots$, by the scheme:

$$
x_{x+1}=F\left(x_{n-p+1}, \cdots, x_{n}\right), \quad n=0,1,2, \cdots
$$

The above scheme will yield a sequence $\left\{x_{n}\right\}, n=0,1,2, \cdots$, if $u_{0}=$ $\left(x_{-p+1}, \cdots, x_{0}\right)$ is an admissible system of starting points in the sense given by the following defintion:

Definition 1 . Consider an operator $F: E \subset E_{1}^{p} \rightarrow E_{1}$ and define recursively

$$
\tilde{E}_{0}=E, \tilde{E}_{n+1}=\left\{u=\left(y_{1}, \cdots, y_{p}\right) \in \tilde{E}_{n} ;\left(y_{2}, \cdots, y_{p}, F(u)\right) \in \tilde{E}_{n}\right\}, \quad n=0,1,2 .
$$


Any $u_{0} \in E_{\infty}=\bigcap_{n \geq 0} \tilde{E}$ will be called an admissible system of starting points for the scheme (4).

If $u_{0}$ is an admissible system of starting points for the scheme (4), we shall say that (4) is well defined.

Definition 2. Let $C$ be a class of pairs $\left(f, v_{0}\right)$ where $f$ is a nonlinear operator defined on a subset $E_{f}$ of a Banach space $E_{1}$ with values in a Banach space $E_{2}$, and $v_{0}=\left(x_{-k+1}, \cdots, x_{0}\right) \in E_{f}^{k}$. Let $p \leq k$. By an iterative procedure of type $(p ; 1)$ for the class $C$, we mean an application which associates with any $\left(f, v_{0}\right) \in C$ an operator $F: E \subset E_{f}^{p} \subset E_{1}$ having the following two properties:

(i) $u_{0}=\left(x_{-p+1}, \cdots, x_{0}\right)$ is an admissible system of starting points for the scheme (4);

(ii) the sequence $\left\{x_{n}\right\}, n=0,1,2, \cdots$ given by (4) converges to a root $x^{*}$ of (1). Having an iterative procedure of type $(p ; 1)$ for the class $C$ it is important to find a function $\alpha: \mathbb{Z}_{+} \rightarrow \mathbb{R}_{+}$and a function $\beta: \mathbb{R}_{+}^{p} \rightarrow \mathbb{R}_{+}$such that the following inequalities are satisfied

$$
\begin{gathered}
d\left(x_{n}, x^{*}\right) \leq \alpha(n) \\
d\left(x_{n}-x^{*}\right) \leq \beta\left(d\left(x_{n-p+1}, x_{n-p}\right), \cdots, d\left(x_{n}, x_{n-1}\right)\right)
\end{gathered}
$$

for every pair $\left(f, x_{0}\right) \in C$ and every positive integer $n$.

The inequalities (5) are called apriori estimates because the right hand side can be computed before obtaining the points $x_{1}, \cdots, x_{n}$ via (4), while the inequalities (3) are called aposteriori estimates because their right hand side can be computed only after obtaining these points.

The estimates (5) and/or (6) will be called sharp if there exists a pair $\left(f, u_{0}\right) \in C$ for which these estimates are attained for all $n=1,2,3, \cdots$.

In the study of (4), we use the nondiscrete mathematical induction. The method was initiated by V. Pták by refining the closed graph theorem [7], [8]. V. Pták used this method to investigate iterative algorithms of type (4) with $p=1$. In [7] the method was extended for any $p$. See also [8] for the history of nondis- 
crete mathematical induction and its application to different gxeneralizations of the secant method.

Here we restate the results obtained in [6]. Let $T$ denote either the set of all positive numbers, or an interval of the form

$$
(0, b]=\{x \in \mathbb{R} ; \quad 0<x \leq b\} .
$$

Let $\omega$ be a mapping of the cartesian product $T^{p}$ into $T$ and let us consider the "iterates" $\omega^{(n)}$ of $\omega$ given for each $t=\left(t_{1}, \ldots, t_{p}\right) \in T^{p}$ by the following scheme:

$$
\omega^{(0)}(t)=t_{p}, \omega^{(n+1)}(t)=\omega^{(n)}\left(t_{2}, \cdots, t_{p}, \omega(t)\right), \quad n=0,1,2, \cdots .
$$

Definition 3. A mapping $\omega: T^{p} \rightarrow T$, with the above iteration law, is called a rate of convergence of type $(p ; 1)$ on $T$, if the series

$$
\sigma(t)=\sum_{n=0}^{\infty} \omega^{(n)}(t)
$$

is convergent for all $t \in T^{p}$.

From now on $F$ will be a mapping of $E$ into $E_{3}$, where $E_{3}$ is a complete metric space, and $E$ a subset of the cartesian product $E_{3}^{p}$. We attach to $F$ the mapping $\bar{F}: E \rightarrow E_{3}^{p}$, defined for every $u=\left(y_{1}, \ldots, y_{p}\right) \in E$ by

$$
\bar{F}(u)=\left(y_{2}, \ldots, y_{p}, F(u)\right) .
$$

Denoting $u_{n}=\left(x_{n-p+1}, \ldots, x_{n}\right)$ we have

$$
u_{n+1}=\bar{F}\left(u_{n}\right), \quad n=0,1,2, \cdots
$$

Similarly, we attach to $\omega$ the mapping $\bar{\omega}: T^{p} \rightarrow T^{p}$ defined by

$$
\bar{\omega}(t)=\left(t_{2}, \ldots, t_{p}, \omega(t)\right), \quad t=\left(t_{1}, \ldots, t_{p}\right) \in T^{p} .
$$

Denote by $\bar{\omega}^{(n)}$ the iterates of $\bar{\omega}$ in the sense of the usual composition of functions, that is

$$
\bar{\omega}^{(0)}(t)=t, \quad \bar{\omega}^{(n+1)}(t)=\bar{\omega}\left(\bar{\omega}^{(n)}(t)\right)
$$


Then (7) becomes

$$
\omega^{(0)}(t)=t_{p}, \quad \omega^{(n+1)}(t)=\omega\left(\bar{\omega}^{(n)}(t)\right)
$$

Finally, we introduce the notation

$$
\beta(t)=\sigma(t)-t_{p}
$$

From (8) and (11) it follows that

$$
\beta(t)=\sigma(\bar{\omega}(t))
$$

With the above notation we can state the following proposition whose proof can be found in [6] or [7].

Proposition 1. Let $E_{3}$ be a complete metric space and let. $E$ be a subset of $E_{3}^{p}$. Let us consider the operators $F: E \rightarrow E_{3}$ and $Z: T^{p} \rightarrow \exp (E)$, where $\exp (E)$ denotes the class of all subsets of $E$. Let $\omega$ be a rate of convergence of type $(p ; 1)$ on $T$.

If there exists $u_{0}=\left(x_{-p+1}, \ldots, x_{0}\right) \in \mathbb{E}$ and $t_{0} \in T^{p}$ such that

$$
u_{0} \in Z\left(t_{0}\right)
$$

and if the relations

$$
\begin{aligned}
& F(u) \in Z(\bar{\omega}(t)) \\
& d\left(F(u), y_{p}\right) \leq t_{p}
\end{aligned}
$$

are satisfied for all $t=\left(t_{1}, \ldots, t_{p}\right) \in T^{p}$ and $u=\left(y_{1}, \ldots, y_{p}\right) \in Z(t)$, then:

(i) the iteration (4) is well defined.

(ii) There exists an $x^{*} \in E_{3}$ such that $x^{*}=\lim _{n \rightarrow \infty} x_{n}$.

(iii) The following relations are satisfied for all $n=0,1,2, \cdots$

$$
\begin{aligned}
& u_{n} \in Z\left(\bar{\omega}^{(n)}\left(t_{0}\right)\right), \\
& d\left(x_{n+1}, x_{n}\right) \leq \omega^{(n)}\left(t_{0}\right), \\
& d\left(x_{n}, x_{0}\right) \leq \sigma\left(t_{0}\right)-\sigma\left(\bar{\omega}^{(n)}\left(t_{0}\right)\right), \\
& d\left(x_{n}, x^{*}\right) \leq \sigma\left(\bar{\omega}^{(n)}\left(t_{0}\right)\right) .
\end{aligned}
$$


(iv) Let $n$ be a positive integer and let $d_{n} \in T^{p}$; if $u_{n-1} \in Z\left(d_{n}\right)$, then

$$
d\left(x_{n}, x^{*}\right) \leq \beta\left(d_{n}\right) .
$$

We will need the definition:

Definition 4. Let $E_{1}$ and $E_{2}$ be two Banach spaces and let $E_{4}$ be a subset of $E_{1}$. Let $f: E_{4} \rightarrow E_{2}$ be a nonlinear operator which is Fréchet differentiable on $E_{4}$. We say that the Fréchet-derivative $f^{\prime}(x)$ is Hölder continuous over $E_{4}$ if for some $c>0$ and $q \in[0,1]$, and all $x, y \in E_{4}$

$$
\left\|f^{\prime}(x)-f^{\prime}(y)\right\| \leq c\|x-y\|^{q} .
$$

In this case, we say $f^{\prime}(\cdot) \in H_{E_{4}}(c, q)$.

Definition 5. Let $E_{1}$ and $E_{2}$ be two Banach spaces and let $E_{4}$ be a convex subset of $E_{1}$. Let $f: E_{4} \rightarrow E_{2}$ be a nonlinear operator which is Fréchetdifferentiable on $E_{4}$. A mapping $\delta f: E_{4} \times E_{4} \rightarrow L\left(E_{1}, E_{2}\right)$, (the space of bounded linear operators from $E_{1}$ to $E_{2}$ ) will be called a consistent generalized approximation of $f^{\prime}$, if there exists a constant $d>0$ such that

$$
\left\|\delta f(x, y)-f^{\prime}(z)\right\| \leq d\left(\|x-z\|^{q}+\|y-z\|^{q}\right), \quad q \in[0,1]
$$

and for all $x, y, z \in E_{4}$.

The above condition implies the Hölder continuity of $f^{\prime}$. Since,

$$
\begin{aligned}
& \left\|f^{\prime}(x)-f^{\prime}(y)\right\|=\left\|\left(f^{\prime}(x)-\delta f(x, y)\right)+\left(\delta f(x, y)-f^{\prime}(y)\right)\right\| \\
\leq & d\left(\|x-x\|^{q}+\|y-x\|^{q}\right)+d\left(\|x-y\|^{q}+\|y-y\|^{q}\right) \\
\leq & 2 d\|x-y\|^{q} .
\end{aligned}
$$

That is

$$
\left\|f^{\prime}(x)-f^{\prime}(y)\right\| \leq c\|x-y\|^{q}, \quad c=2 d \text { and for all } x, y \in E_{4} .
$$

Also, as in [1], we can easily show that

$$
\left\|f(x)-f(y)-f^{\prime}(x)(x-y)\right\| \leq \frac{c}{1+q}\|x-y\|^{1+q}
$$


for all $x, y \in E_{4}$.

Finally, for all $x, y, u, v \in E_{4}$ we have

$$
\begin{aligned}
& \|f(u)-f(v)-\delta f(x, y)(u, v)\| \\
= & \left\|\left(f(u)-f(v)-f^{\prime}(v)(u-v)\right)+\left(f^{\prime}(v)-\delta f(x, y)\right)(u-v)\right\| \\
\leq & \frac{2 d}{1+q}\|u-v\|^{1+q}+d\left(\|x-v\|^{q}+\|y-v\|^{q}\right)\|u-v\| \\
\leq & d\left(\frac{2}{1+q}\|u-v\|^{q}+\|x-v\|^{q}+\|y-v\|^{q}\right)\|u-v\| .
\end{aligned}
$$

Let $C\left(h_{0}, q_{0} ; r_{0}\right)$ be the class of all triplets $\left(f, x_{0}, x_{-1}\right)$ satisfying the following properties:

$\left(P_{1}\right) f$ is a nonlinear operator having the domain of definition $E_{f}$ included into a Banach space $E_{1}$ and taking values in a Banach space $E_{2}$.

$\left(P_{2}\right) x_{0}$ and $x_{-1}$ are two points of $E_{f}$ such that

$$
0<\left\|x_{0}-x_{-1}\right\| \leq q_{0},\left\|x_{0}-x_{-1}\right\|<\mu .
$$

$\left(P_{3}\right) f$ is Fréchet-differentiable in the open ball

$$
U=U\left(x_{0}, \mu\right)=\left\{x \in E_{f} /\left\|x-x_{0}\right\| \leq \mu\right\}
$$

and continuous on its closure $\bar{U}$.

$\left(P_{4}\right)$ there exists a consistent generalized approximation $\delta f$ of $f^{\prime}$ such that $D_{0}:=\delta f\left(x_{-1}, x_{0}\right)$ is invertible and

$$
\| D_{0}^{-1}\left(\delta f(x, y)-f^{\prime}(z) \| \leq h_{0}\left(\|x-z\|^{q}+\|y-z\|^{q}\right)\right.
$$

for all $x, y, z \in U$ and some $h_{0} \geq d \cdot\left\|D_{0}^{-1}\right\|$.

$\left(P_{5}\right)$ the following inequality is satisfied:

$$
\left\|D_{0}^{-1} f\left(x_{0}\right)\right\| \leq \bar{r}_{0} .
$$

$\left(P_{6}\right)$ Assume that for each fixed $q \in[0,1]$, there exists a minimum positive number $\mu_{0}$ such that the following conditions hold:

$$
\alpha_{0}=\frac{h_{0} r_{0}^{q}(3+q)}{(1+q)\left[1-h_{0}\left(2 \mu_{0}^{q}+q_{0}^{q}\right)\right]}<1, \quad r_{0}=\max \left\{\bar{r}_{0}, q_{0}\right\}
$$




$$
u_{0}=\frac{r}{1-\alpha_{0}}
$$

and

$$
h_{0}\left(\frac{4}{q+1} \mu_{0}^{q}+q_{0}^{q}\right)<1 .
$$

Define also the functions $\omega$ and $\sigma$ on $T=\left[0, \mu_{0}\right]$ by

$$
\omega(r)=\alpha_{0} r
$$

and

$$
\sigma(r)=\frac{r}{1-\alpha_{0}}
$$

We will use the estimate

$$
\begin{aligned}
& \left\|D_{0}^{-1}(f(u)-f(v)-\delta f(x, y)(u-v))\right\| \\
= & \| D_{0}^{-1}\left(f(u)-f(v)-f^{\prime}(v)(u-v)+D_{0}^{-1}\left(f^{\prime}(v)-\delta f(x, y)\right)(u-v) \|\right. \\
\leq & \frac{2 d\left\|D_{0}^{-1}\right\|}{1+q}\|u-v\|^{1+q}+h_{0}\left(\|x-v\|^{q}+\|y-v\|^{q}\right)\|u-v\|
\end{aligned}
$$

(by (24) and (26))

$$
\leq h_{0}\left[\frac{2}{1+q}\|u-v\|^{q}+\|x-v\|^{q}+\|y-v\|^{q}\right]\|u-v\| .
$$

\section{Main results}

Using (2) we shall show that if $\left(f, x_{0}, x_{-1}\right) \in C\left(h_{0}, q_{0}, r_{0}\right)$ then (1) has a solution $x^{*}$ which is unique in a certain neighborhood of $x_{0}$.

We will need the following lemma whose proof can be obtained immediately by using relation (8).

Lemma 1. The function $\omega$ given by (31) is a rate of convergence of type $(1,1)$ on the interval $T=\left(0, \mu_{0}\right)$ and the corresponding $\sigma$-function is given by (32).

We will now prove the main result.

Theorem 1. If $\left(f, x_{0}, x_{-1}\right) \in C\left(h_{0}, q_{0}, r_{0}\right)$, then 
(a) the sequence $\left\{x_{n}\right\}, n=-1,0,1,2, \cdots$ is well defined on $U=U\left(x_{0}, \mu_{0}\right)$, where $\mu_{0}=\sigma\left(r_{0}\right)$ remains in $U$ and converges to a solution $x^{*}$ of equation (1) which is unique in $U\left(x_{0}, \mu_{0}\right), \mu_{0}<\mu$. Moreover, the following estimates are true:

$$
\begin{gathered}
\left\|x_{n+1}-x^{*}\right\| \leq \sigma\left(\omega^{(n)}\left(r_{0}\right)\right), \quad n=0,1,2, \cdots \\
\left\|x_{n}-x^{*}\right\| \leq \sigma\left(\left\|x_{n}-x_{n-1}\right\|\right)-\left\|x_{n}-x_{n-1}\right\|, \quad n=0,1,2, \cdots
\end{gathered}
$$

where $\omega, \sigma$ are given by (31) and (32) respectively.

Proof. Let $A=\left\{u=(y, x) \in U^{2} ; \delta f(y, x)\right.$ is invertible $\}$ and $F: A \rightarrow E_{1}$ be a mapping such that

$$
F u=x-\delta f(y, x)^{-1} f(x) .
$$

Set $t_{0}=\left(q_{0}, r_{0}\right)$. Then, by (31) and (32), $\sigma\left(t_{0}\right)=\mu_{0}$. Define the set

$$
Z(t)=\left\{u=(y, x) \in E_{1}^{2} ; y \in U,\|x-y\| \leq q,\left\|x-x_{0}\right\| \leq \mu_{0}-\sigma(t),\right.
$$

$t=(q, r) \in T^{2}$, the linear operator $D=\delta f(y, x)$ is invertible and $\left\|D^{-1} f(x)\right\| \leq$ $r\}$.

Then, we can immediately get $Z(t) \leq A$ and $u_{0}=\left(x_{-1}, x_{0}\right) \in Z\left(t_{0}\right)$. We will now show that if $u=(y, x) \in Z(t)$, then $(x, F u) \in Z(\omega(t))$. We denote $z=F u$ and we will show that

$$
\begin{aligned}
& x \in U,\|z-x\| \leq r, \\
& \left\|z-x_{0}\right\| \leq \mu_{0}-\sigma(\omega(t)),
\end{aligned}
$$

the operator $D_{1}=\delta f(x, z)$ is invertible and

$$
\left\|D_{1}^{-1} f(z)\right\| \leq \omega(t)
$$

Since $z-x=-D^{-1} f(x)$, estimate (37) follows at once. Then we have

$$
\left\|z-x_{0}\right\| \leq\|z-x\|+\left\|x-x_{0}\right\| r+\mu_{0}-\sigma(t)=\mu_{0}-\sigma(\omega(t)),
$$


since $\sigma(\omega(t))=\sigma(t)-r$.

By (33) we have

$$
\begin{aligned}
\left\|D_{0}^{-1}\left(D_{0}-D_{1}\right)\right\| & \leq\left\|D_{0}^{-1}\left(D_{0}-f^{\prime}\left(x_{0}\right)\right)\right\|+\left\|D_{0}^{-1}\left(f^{\prime}\left(x_{0}\right)-D_{1}\right)\right\| \\
& \leq h_{0}\left(\left\|x_{0}-x_{-1}\right\|^{q}+\left\|x-x_{0}\right\|^{q}+\left\|z-x_{0}\right\|^{q}\right) \\
& \leq h_{0}\left(2 \mu_{0}^{q}+q_{0}^{q}\right)<1, \quad \text { by }(30) .
\end{aligned}
$$

By the Banach lemma on invertible operators

$$
\left\|D_{1}^{-1} D_{0}\right\| \leq \frac{1}{1-h_{0}\left(2 \mu_{0}^{q}+q_{0}^{q}\right)}
$$

Also, from (36) we obtain the approximation

$$
f(x)=f(z)-f(x)-\delta f(y, x)(z-x),
$$

and by (33) we get

$$
\left\|D_{0}^{-1} f(z)\right\| \leq h_{0}\left(\frac{2}{1+q}\|z-x\|^{q}+\|x-y\|^{q}\right)\|z-x\| .
$$

By $(31),(40)$ and (41) we now obtain

$$
\left\|D_{1}^{-1} f(z)\right\|=\left\|\left(D_{0}^{-1} D_{1}\right)^{-1} D_{0}^{-1} f(z)\right\| \leq \omega(t) .
$$

The validity of (37), (38) and (39) has now been verified. It now follows that hypotheses (13), (14) and (15) of Proposition 1 are satisfied. Hence, the sequence (2) converges to a point $x^{*} \in A$ and estimates (34) are satisfied. Moreover, we have

$$
\begin{aligned}
& \left(x_{n-2}, x_{n-1}\right) \in Z\left(\omega^{(n-1)}\left(t_{0}\right)\right), \quad n \geq 1 \\
& \left\|x_{n+1}-x_{n}\right\| \leq \omega^{(n)}\left(t_{0}\right), \quad n \geq 0 .
\end{aligned}
$$

The function $\sigma$ being increasing (in the sense that $q_{1} \leq q_{2}$ are $r_{1} \leq r_{2}$ implies $\sigma\left(q_{1}, r_{1}\right) \leq \sigma\left(q_{2}, r_{2}\right)$, from the above, one can deduce that

$$
\left(x_{n-2}, x_{n-1}\right) \in Z\left(\left\|x_{n-1}-x_{n-2}\right\|,\left\|x_{n}-x_{n-1}\right\|\right), \quad n \geq 1,
$$

from which estimate (58) follows. 
Set $t=\omega^{(n)}\left(t_{0}\right), z=x_{n+1}$ in (41) and let $n$ tend to infinity. Then $f\left(x^{*}\right)=0$, which shows that $x^{*}$ is a solution of equation (1). To show uniqueness, we assume that there exists another solution $y^{*}$ of equation (1) in $U\left(x_{0}, \mu_{0}\right)$. Then using (26), we get

$$
\begin{aligned}
& \int_{0}^{1}\left\|D_{0}^{-1}\left[f^{\prime}\left(y^{*}+t\left(x^{*}-y^{*}\right)\right)-D_{0}\right]\right\| d t \\
\leq & \int_{0}^{1}\left\|D_{0}^{-1}\left[f^{\prime}\left(y^{*}+t\left(x^{*}-y^{*}\right)\right)-\delta f\left(x_{0}, x_{0}\right)\right]\right\| d t+\left\|D_{0}^{-1}\left(\delta f\left(x_{0}, x_{0}\right)-D_{0}\right)\right\| \\
\leq & h_{0}\left[2 \int_{0}^{1}\left((1-t)^{q}\left\|y^{*}-x_{0}\right\|^{q}+t^{q}\left\|x^{*}-x_{0}\right\|^{q}\right) d t+q_{0}^{q}\right] \\
\leq & h_{0}\left(\frac{4}{q+1} \mu_{0}^{q}+q_{0}^{q}\right)<1, \quad \text { by }(30) .
\end{aligned}
$$

Hence, the linear operator $\int_{0}^{1} f^{\prime}\left(y^{*}+t\left(x^{*}-y^{*}\right)\right) d t$ is invertible. From the approximation

$$
f\left(x^{*}\right)-f\left(y^{*}\right)=\int_{0}^{1} f^{\prime}\left(y^{*}+t\left(x^{*}-y^{*}\right)\right)\left(x^{*}-y^{*}\right) d t
$$

it now follows that $x^{*}=y^{*}$, which completes the proof of the theorem.

Remark 1. The case $q=1$ has been investigated already in the elegant work contained in [5] under different hypotheses. The main reason for our study is to cover cases when $q \in[0,1)$, which are not covered in [5].

Remark 2. Let us consider the scalar equation

$$
g(t)=0
$$

where

$$
\begin{gathered}
g(t)=\beta_{0} t^{1+q}+\beta_{1} t+\beta_{2} t^{q}+\beta_{3}, \\
\beta_{0}=2 h_{0}(1+q), \quad \beta_{1}=-2 h_{0} r_{0}(1+q), \\
\beta_{2}=h_{0}(3+q) r_{0}^{q}-(1+q)\left(1-h_{0} q_{0}^{q}\right)
\end{gathered}
$$


and

$$
\beta_{3}=r_{0}(1+q)\left(1-h_{0} q_{0}^{q}\right) .
$$

Then, by (29) we have

$$
g\left(\mu_{0}\right)=0 .
$$

We can now provide sufficient conditions for the existence of two positive solutions of equation $g(t)=0$.

Theorem 2. Assume that the following conditions are satisfied:

(a) $\beta_{1}+\beta_{2}<0 ; g(0)>0$;

(b) $\left(\beta_{1}+\beta_{2}\right)^{2}-4 \beta_{0} \beta_{3}>0$,

and

(c) $g\left(\frac{\beta_{1}+\beta_{2}}{2 \beta_{0}}\right)<0$.

Then there exists a minimum positive solution $\mu_{0}$ of equation

$$
g(t)=0
$$

Proof. The function $h(t)=\beta_{0} t^{2}+\left(\beta_{1}+\beta_{2}\right) t+\beta_{3}$ has a minimum at $t_{m}=-\frac{\beta_{1}+\beta_{2}}{2 \beta_{0}}$. Hypotheses (a) and (b) imply that the equation $h(t)=0$ has two positive solutions. By hypothesis $(\mathrm{c}), g\left(t_{m}\right)<0$. Since $g(t)$ is continuous, $g(0)>0$ and $g(t)>0$ for $t$ sufficiently large, we conclude that $g(t)$ has two positive solutions $\mu_{0}$ and $\mu_{1}$ of which we choose the minimum on as our $\mu_{0}$.

The proof of the theorem is now complete.

Remark 3. Under the hypotheses of Theorems 1 and 2, by using (42) the uniqueness of the solution $x^{*}$ of equation (1) can be extended to the ball $\bar{U}\left(x_{0}, \mu_{1}\right)$. The existence of $\mu_{1}$ is guaranteed by Theorem 2 . To achieve this goal we just need to replace the existence condition (30) by

$$
h_{0}\left[\frac{2}{q+1}\left(\mu_{0}^{q}+\mu_{1}^{q}\right)+q_{0}^{q}\right]<1 .
$$

We can then also set $\mu=\mu_{1}$ in Theorem 1 . 


\section{Applications}

Example 1. Consider the function $G$ defined on $[0, b]$ by

$$
G(t)=\frac{2}{3} t^{\frac{3}{2}}+t-3
$$

for some $b>0$.

Let $\|$ || denote the $\max$ norm on $\mathbb{R}$, then

$$
\left\|G^{\prime \prime}(t)\right\|=\max _{t \in[0, b]}\left|\frac{1}{2} t^{-\frac{1}{2}}\right|=\infty
$$

which implies that the basic hypothesis in [3] (or the Lipschitz continuity of $f^{\prime}$ for $q \neq 1$ in [5]) for the application of Newton's method is not satisfied for finding a solution of the equation

$$
G(t)=0 .
$$

However, it can easily be seen that $G^{\prime}(t)$ is Hölder continuous on $[0, b]$ with

$$
c=1 \text { and } q=\frac{1}{2} \text {. }
$$

Therefore, under the assumptions of Theorem 1, iteration (2) will converge to a solution $t^{*}$ of equation (43).

Another interesting application for Theorem 1 is given by the following example.

Example 2. Consider the differential equation

$$
\begin{gathered}
x^{\prime \prime}+x^{1+q}=0, \quad q \in[0,1] \\
x(0)=x(1)=0 .
\end{gathered}
$$

We divide the interval $[0,1]$ into $n$ subintervals and we set $h=\frac{1}{n}$. Let $\left\{v_{k}\right\}$ be the points of subdivision with

$$
0=v_{0}<v_{1} \ldots<v_{n}=1
$$


A standard approximation for the second derivative is given by

$$
x_{i}^{\prime \prime}=\frac{x_{i-1}-2 x_{i}+x_{i+1}}{h^{2}} ; \quad x_{i}=x\left(v_{i}\right), \quad i=1,2, \cdots, n-1 .
$$

Take $x_{0}=x_{n}=0$ and define the operator $\tilde{F}: \mathbb{R}^{n-1} \rightarrow \mathbb{R}^{n-1}$ by

$$
\begin{aligned}
& \tilde{F}(x)=H(x)+h^{2} \varphi(x)
\end{aligned}
$$

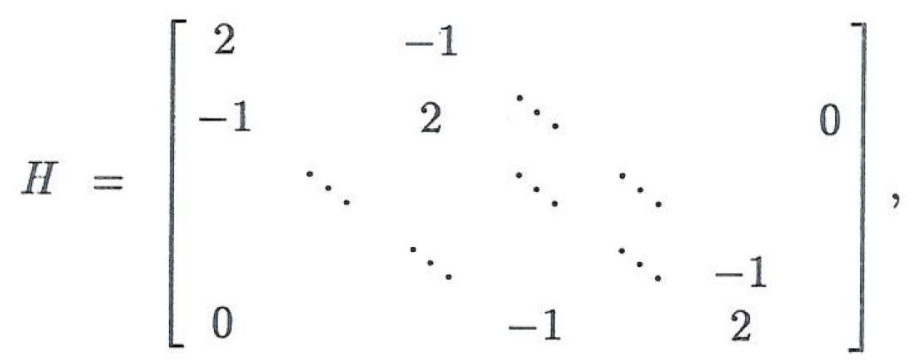

$$
\begin{aligned}
& \varphi(x)=\left[\begin{array}{c}
x_{1}^{1+q} \\
x_{2}^{1+q} \\
\vdots \\
x_{n-1}^{1+q}
\end{array}\right],
\end{aligned}
$$

and

$$
x=\left[\begin{array}{c}
x_{1} \\
x_{2} \\
\vdots \\
x_{n-1}
\end{array}\right]
$$

Then

$$
\tilde{F}^{\prime}(x)=H+h^{2}(q+1)\left[\begin{array}{cccc}
x_{1}^{q} & & & 0 \\
& x_{2}^{q} & & \\
& & \ddots & \\
0 & & & x_{n-1}^{q}
\end{array}\right] .
$$

Newton's method cannot be applied to the equation

$$
\tilde{F}(x)=0 \text {. }
$$

We may not be able to evaluate the second Fréchet-derivative since it would involve the evaluation of quantities of the form $x_{i}^{-p}$ and they may not exist.

We will face the same difficulty in verifying the Lipschitz continuity of $\tilde{F}^{\prime}$. 
Let $x \in \mathbb{R}^{n-1}, H \in \mathbb{R}^{n-1} \times \mathbb{R}^{n-1}$ and define the norms of $x$ and $H$ by

$$
\begin{gathered}
\|x\|=\max _{1 \leq j \leq n-1}\left|x_{j}\right| \\
\|H\|=\max _{1 \leq j \leq n-1} \sum_{k=1}^{n-1}\left|h_{j k}\right| .
\end{gathered}
$$

For all $x, z \in \mathbb{R}^{n-1}$ for which $\left|x_{i}\right|>0,\left|z_{i}\right|>0, i=1,2, \cdots, n-1$ we obtain, for $q=\frac{1}{2}$ say,

$$
\begin{aligned}
\left\|\tilde{F}^{\prime}(x)-\tilde{F}^{\prime}(z)\right\| & =\left\|\operatorname{diag}\left\{\left(1+\frac{1}{2}\right) h^{2}\left(x_{j}^{\frac{1}{2}}-z_{j}^{\frac{1}{2}}\right)\right\}\right\| \\
& =\frac{3}{2} h^{2} \max _{1 \leq j \leq n-1}\left|x_{j}^{\frac{1}{2}}-z_{j}^{\frac{1}{2}}\right| \leq \frac{3}{2} h^{2}\left[\max \left|x_{j}-z_{j}\right|\right]^{\frac{1}{2}} \\
& =\frac{3}{2} h^{2}\|x-z\|^{\frac{1}{2}} .
\end{aligned}
$$

A linear operator $L \in L\left(\mathbb{R}^{n-1}, R^{n-1}\right)$ can be represented by a matrix with entries $q_{i j}$ and

$$
\|L\|=\max \left\{\sum_{j=1}^{n-1}\left|q_{i j}\right| ; 1 \leq i \leq n-1\right\} .
$$

Let us denote by $\tilde{F}_{1}, \ldots, \tilde{F}_{n-1}$ the components of $\tilde{F}$. For each $v \in \mathbb{R}^{n-1}$ we can write

$$
\tilde{F}(v)=\left(\tilde{F}_{1}(v), \ldots, \tilde{F}_{n-1}(v)\right)^{t r} .
$$

Let $v, w \in \mathbb{R}^{n-1}$ and define $\delta \tilde{F}(v, w)$ by the matrix with entries

$$
\begin{aligned}
\delta \tilde{F}(v, w)_{i j}= & \frac{1}{v_{j}-w_{j}}\left(\tilde{F}_{i}\left(v_{1}, \ldots, v_{j}, w_{j+1}, \ldots, w_{m}\right)\right. \\
& \left.-\tilde{F}_{i}\left(v_{1}, \ldots, v_{j-1}, w_{j}, \ldots, w_{m}\right)\right), m=n-1 .
\end{aligned}
$$

It can easily be seen that the operator defined by $(47)$ satisfies $\delta \tilde{F}(v, w) \in$ $L\left(\mathbb{R}^{n-1}, \mathbb{R}^{n-1}\right)$.

Denote by

$$
P_{j} \tilde{F}_{i}(v)=\frac{\partial \tilde{F}_{i}(v)}{\partial v_{j}}, \quad i, j=1,2, \ldots, n-1
$$


We can choose $n=10$ which gives (9) equations for iteration (2), if we look at it as a system of linear equations given $z_{-1}, z_{0} \in \mathbb{R}^{9}$. Since a solution would vanish at the end points and be positive in the interior a reasonable choice of initial approximation seems to be $130 \sin \pi x$. This gives us the following vector

$$
z_{-1}=\left[\begin{array}{ll}
4.015241 & E+01 \\
7.637852 & E+01 \\
1.051351 & E+02 \\
1.236112 & E+02 \\
1.299991 & E+02 \\
1.236752 & E+02 \\
1.052571 & E+02 \\
7.654622 & E+01 \\
4.034951 & E+01
\end{array}\right]
$$

Choose $z_{0}$ by setting

$$
z_{0}\left(v_{i}\right)=z_{-1}\left(v_{i}\right)-10^{-5}, i=1,2, \cdots, 9 .
$$

Using iteration (26) with the above values and (32), after seven iterations we get

$$
z_{6}=\left[\begin{array}{ll}
3.357455 & E+01 \\
6.520294 & E+01 \\
9.156631 & E+01 \\
1.091680 & E+02 \\
1.153630 & E+02 \\
1.091680 & E+02 \\
9.156663 & E+02 \\
6.520294 & E+01 \\
3.357455 & E+01
\end{array}\right] \text { and } z_{7}=\left[\begin{array}{l}
3.357450 E+01 \\
6.520290 E+01 \\
9.156660 E+01 \\
1.091680 E+02 \\
1.536301 E+02 \\
1.091680 E+02 \\
9.156660 E+02 \\
6.520290 E+01 \\
3.357450 E+01
\end{array}\right]
$$

We choose $z_{6}=x_{-1}$ and $z_{7}=x_{0}$ for our Theorem 1. From now on we assume that $\tilde{F}$ is restricted on $\bar{U}\left(x_{0}, \mu_{0}\right), \mu_{0}=10^{-4}$. With the notation of Theorem 1 we can easily obtain the following results:

$$
\begin{aligned}
& \left\|D_{0}^{-1}\right\|=25.5882 \\
& q=\frac{1}{2}
\end{aligned}
$$




$$
\begin{aligned}
& d=.03, \\
& q_{0}=5.10^{-5}, \\
& \bar{r}_{0}=r_{0}=9.15311 .10^{-5},
\end{aligned}
$$

and

$$
h_{0}=.767646 \text {. }
$$

All the hypotheses of Theorem 1 are now satisfied with the above values. Therefore, the iteration generated by (2) converges to solution $z^{*}$ of equation (46), which is unique in $\bar{U}\left(x_{0}, \mu_{0}\right)$.

Finally, note that the results in [3]-[9] cannot be applied here.

\section{References}

[1] I. K. Argyros, "On the secant method and fixed points of nonlinear equations", Monatshefte fur Mathematik, 106 (1988), 85-94.

[2] H. T. Davis, "Introduction to nonlinear differential and integral equations", Dover Publ., New York, 1962.

[3] L. V. Kantorovich and G. P. Akilov, "Functional analysis in normed spaces", Oxford, Pergamon Press, 1964.

[4] J. M. Ortega and W. C. Rheinboldt, "Iterative solution of nonlinear equations in several variables", Academic Press, New York, 1970.

[5] F. A. Potra, "On a modified secant method. L'analyse Numerique et la theorie de l'approximation", Tome 8, No. 2 (1979), 203-214.

[6] F. A. Potra, "An error analysis of the Secant method", Numer. Math., 38 (1982), 427-445.

[7] F. A. Potra and V. Ptak, "Nondiscrete induction and iterative processes", Pitman Publ., 1984.

[8] V. Ptak, "Nondiscrete mathematical induction. In: General topology and its relations to modern analysis and algebra IV.", Lecture Notes in Mathematics 609, pp. 166-178. Berlin-Heidelberg New York: Springer 1977.

[9] W. C. Rheinboldt, "A unified convergence theory for a class of iterative processes", SIAM J. Numer. Anal., Vol. 5, 1, (1968), 42-63. 